Mappemonde

Revue trimestrielle sur l'image géographique et les formes du territoire

$121 \mid 2017$

Varia

\title{
De la crise urbaine à la réappropriation environnementale et alimentaire du territoire
}

Flaminia Paddeu

\section{OpenEdition}

Journals

Édition électronique

URL : http://journals.openedition.org/mappemonde/3791

DOI : 10.4000/mappemonde.3791

ISSN : 1769-7298

Éditeur

UMR ESPACE

Référence électronique

Flaminia Paddeu, « De la crise urbaine à la réappropriation environnementale et alimentaire du territoire », Mappemonde [En ligne], 121 | 2017, mis en ligne le 01 juillet 2017, consulté le 15 septembre 2020. URL : http://journals.openedition.org/mappemonde/3791

Ce document a été généré automatiquement le 15 septembre 2020

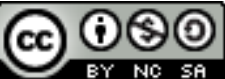

La revue Mappemonde est mise à disposition selon les termes de la Licence Creative Commons Attribution - Pas d'Utilisation Commerciale - Partage dans les Mêmes Conditions 4.0 International. 


\title{
De la crise urbaine à la réappropriation environnementale et alimentaire du territoire
}

\author{
Flaminia Paddeu
}

Références de la thèse

PADDEU F. (2015). De la crise urbaine à la réappropriation du territoire. Mobilisations

civiques pour la justice environnementale et alimentaire dans les quartiers défavorisés de

Detroit et du Bronx à New York. Thèse de doctorat en géographie, Université Paris 4.

1 "Déserts alimentaires ", " environnements obésogènes ", " quartiers au faible potentiel piétonnier »- autant de nouvelles manières de nommer les quartiers défavorisés aux États-Unis. Dans ces inner cities, les habitants relatent un quotidien fait d'efFLuves nauséabonds, d'air pollué, de rivières souillées, de terrains vagues, de squares dégradés et de commerces d'alcool en guise de supermarchés. Les problèmes traditionnels des « ghettos » se doublent ainsi d'inégalités environnementales et alimentaires. Face à ces inégalités, les habitants, via des associations locales, se mobilisent pour améliorer leur environnement quotidien et créer parcs, coulées vertes et fermes urbaines.

2 À partir d'enquêtes de terrain menées dans deux quartiers défavorisés, Hunts Point dans le South Bronx à New York et JefFerson-Mack à Detroit, ma recherche s'est structurée autour des bénéfices territoriaux des mobilisations civiques dans des quartiers en déclin et du potentiel de stratégies environnementales et alimentaires à aborder des enjeux également sociaux, économiques, politiques ou raciaux.

La méthodologie utilisée a privilégié une approche ethnogéographique, à la fois compréhensive et critique, partant des valeurs qui fondent l'action et des systèmes qui contraignent les acteurs. Tous les entretiens menés avec les habitants et militants ont commencé par une discussion portant sur les représentations de l'environnement urbain local, afin d'identifier la gamme de symptômes susceptibles de susciter un engagement focalisé sur les enjeux environnementaux et alimentaires. Cette enquête a été soutenue par un travail d'observation participante et non-participante dont 
rendent compte les clichés photographiques présentés ici: ils donnent à voir la reconfiguration territoriale des espaces vacants dans un quartier de l'East Side de Detroit suite à des mobilisations citoyennes.

\section{Une crise urbaine de l'habiter : désordres, fardeaux environnementaux et accessibilité diminuée}

4 Un premier ensemble de clichés photographiques expose les paysages ainsi que les conditions de vie qui résultent d'une crise urbaine structurelle (Sugrue, 1996). Dans ma démarche, j'ai privilégié une approche fondée sur l'expérience sensible de l'environnement urbain de proximité, qui est apparu pour les enquêté(e)s comme un marqueur d'inhospitalité et d'injustice spatiale. J'ai ainsi mis en évidence que les habitants de ces quartiers subissent une crise urbaine de l'habiter, dans laquelle la concaténation entre les désordres, les nuisances et pollutions ainsi que le manque d'accessibilité aux ressources environnementales et alimentaires est déterminante pour comprendre l'essor des mobilisations.

5 Comme l'illustre cette photographie (figure 1), les conditions de production du quartier en déclin aux États-Unis sont à l'origine de « désordres » (Skogan, 1990) qui afFectent l'environnement matériel (maisons abandonnées aux portes et fenêtres murées, porches qui s'efFondrent, chaussée mal entretenue) comme naturel (friches couvertes de hautes herbes, arbres et buissons non taillés, pelouses inégalement tondues, mauvaises herbes sur les trottoirs, bas-côtés couverts de gravats et de détritus). Ces désordres y sont rendus partiellement responsables du sentiment de vulnérabilité des habitants, accusés de favoriser usages informels ou marginaux (squat, trafic de drogue, stockage de matériaux ou de métaux récupérés, décharges sauvages) ainsi que certains risques (efFondrement, incendies accidentels, criminels ou «ludiques »). 
Figure 1. Les stigmates paysagers de la vacance foncière et immobilière (East Side, Detroit MI)

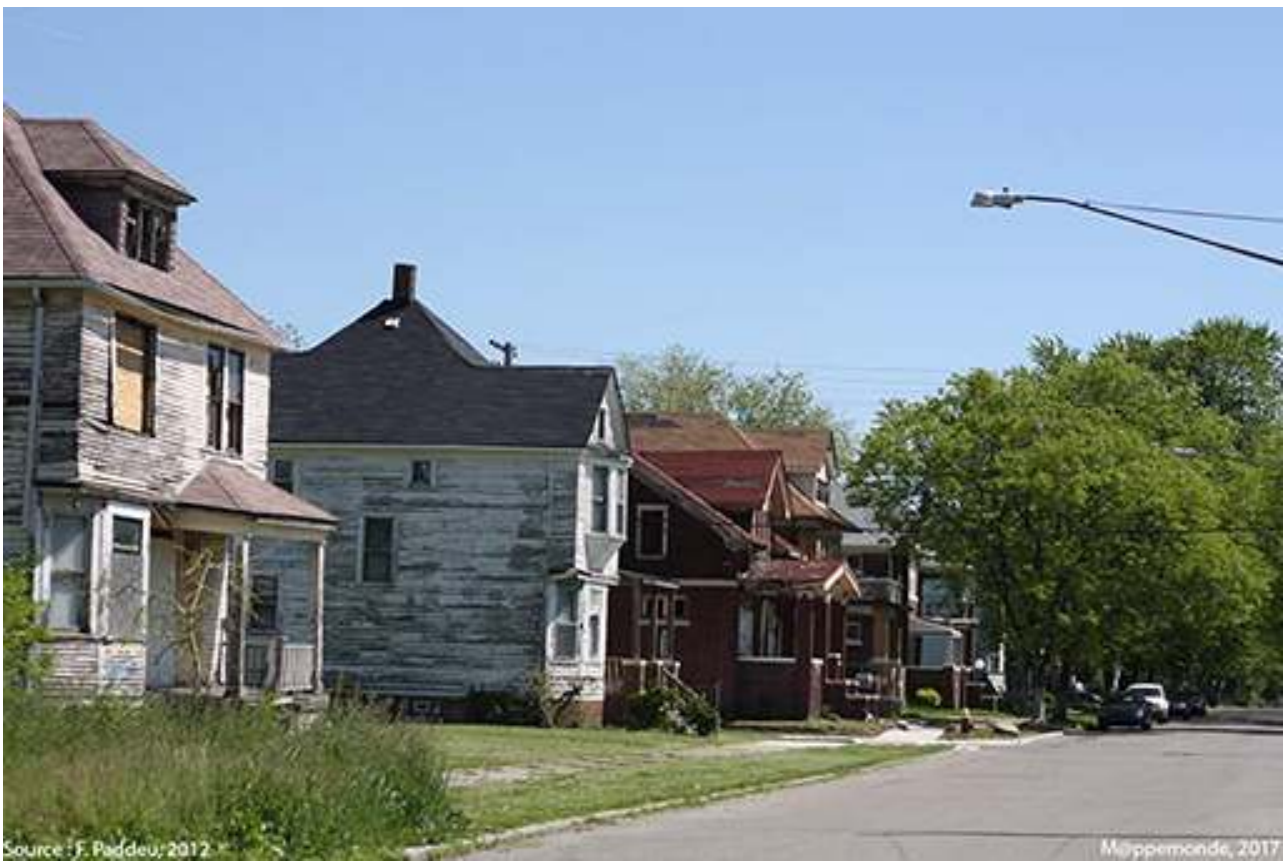

Source : F. Paddeu, 2012

6 Outre ces dégradations, les quartiers en déclin sont caractérisés par une accessibilité diminuée à un certain nombre de ressources (emplois, services de santé, infrastructures éducatives et culturelles), dont les aménités environnementales et les lieux d'approvisionnement alimentaire. Elle est liée à une différenciation entre individus plus ou moins riches ou mobiles, mais aussi à une inégale distribution spatiale des ressources. La photographie ci-dessus (figure 1) illustre ainsi le paysage du « désert alimentaire ", un quartier sans accès facile à des aliments frais, sains et abordables. Au lieu de supermarchés et d'épiceries, les habitants disposent d'un accès alimentaire inexistant ou limité aux fast-foods, épiceries de dépannage, stations-essence ou commerces d'alcool, contribuant à l'augmentation des taux d'obésité et autres maladies nutritionnelles. L'indigence du système de transports en commun à Detroit ainsi que le faible taux de motorisation dans ces quartiers grèvent aussi les capacités d'accessibilité.

\section{L'opportunité de la vacance foncière}

7 Les quartiers en déclin reflètent la distribution spatiale de la valeur foncière. À Detroit, environ un tiers de la ville est constitué d'espaces vacants, soit environ 104000 parcelles inhabitées ou inoccupées, dont $83 \%$ sont en zonage résidentiel (Detroit Works, 2012). Comme le donne à voir la carte que j'ai réalisée (figure 2), certaines zones très touchées par le déclin connaissant un taux de vacance foncière supérieur à $50 \%$ - représentées en jaune -, couvrent $17 \%$ du territoire de Detroit. Le taux de vacance foncière a été calculé par secteur de recensement par un organisme local de cartographie (Data Driven Detroit), dans l'objectif de localiser les zones de concentration de la vacance foncière. Ces zones de haute-vacance forment un hémicycle qui entoure le centre-ville commercial, administratif et universitaire, auquel s'ajoutent de nombreux isolats dispersés. JeFFerson-Mack, le quartier de l'East Side que 
j'ai étudié, fait partie de ces zones où les friches et maisons abandonnées sont devenues majoritaires et caractérisent le paysage urbain.

Figure 2. Les parcelles résidentielles vacantes à Detroit (2000)

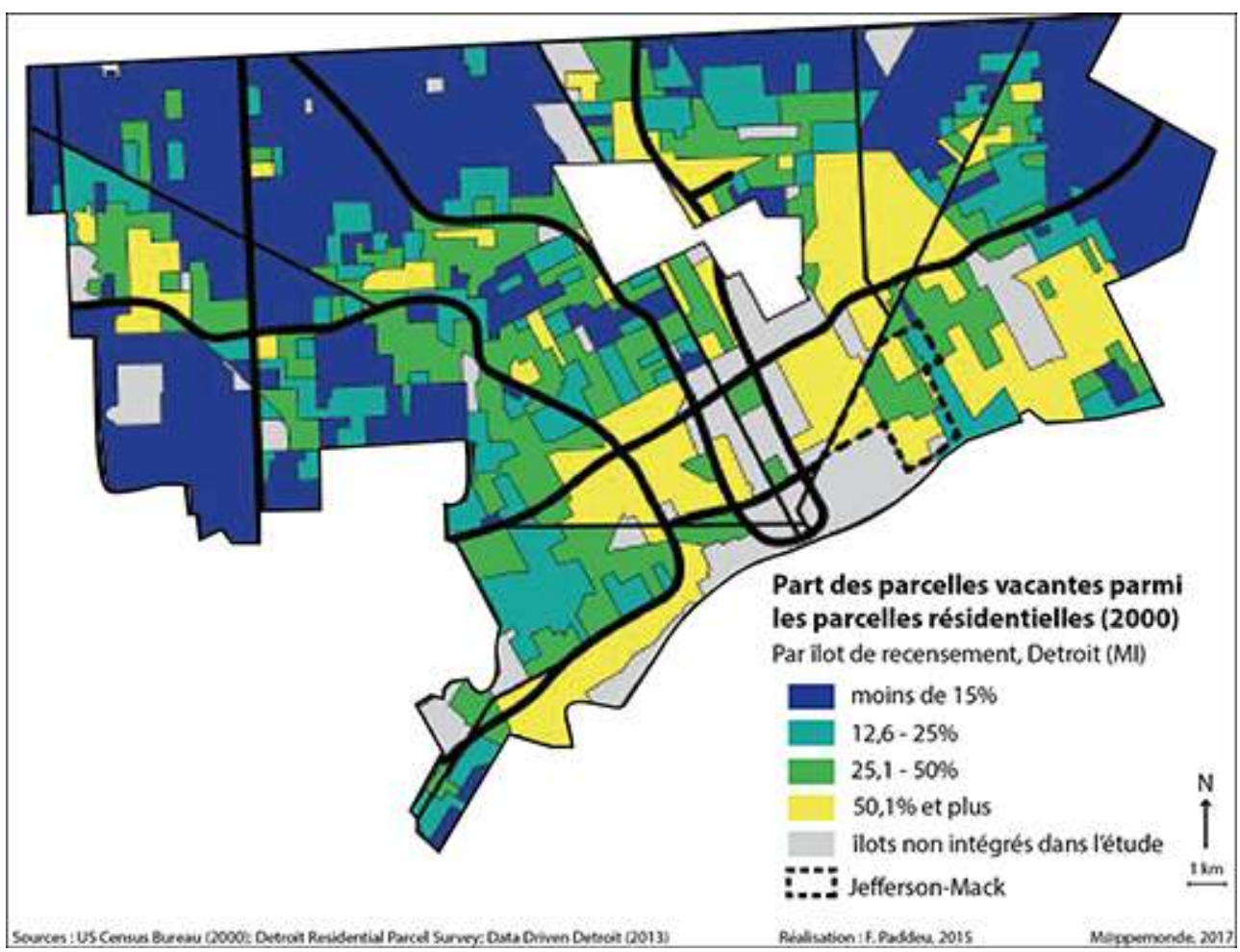

Réalisation : F. Paddeu, 2015

\section{L'agriculture urbaine comme modalité d'une réappropriation multifonctionnelle du territoire}

8 Un second ensemble de clichés vise alors à rendre compte des processus territoriaux de récupération puis de réaménagement des espaces vacants par des groupes d'habitants ou des associations de quartier pour aborder les problèmes identifiés.

Le jardin communautaire du Georgia Street Community Collective (figure 3) est l'une des modalités variées de réappropriation des espaces vacants permettant d'en illustrer la multifonctionnalité (Duchemin et al., 2010). Cette organisation à but non lucratif, fondée en 2002 par un habitant afro-américain du quartier, s'est informellement approprié cinq parcelles inoccupées jouxtant leur local associatif. Les bénéfices alimentaires, notamment l'augmentation de la sécurité, l'accessibilité, la qualité et la durabilité alimentaires, constituent les premiers bénéfices recherchés. Les cultures de laitue, de chou kale, de brocoli, de courge squash ou encore d'okra que l'on voit au premier plan sont ainsi destinées à être vendues directement à la "ferme ", données gratuitement ou autoconsommées dans le cadre des événements de l'association. Le choix des semis est efFectué dans le but de cultiver des légumes caractéristiques du régime alimentaire traditionnel des Afro-américains. Derrière le centre, une autre parcelle est occupée par un poulailler et un enclos avec quelques chèvres, afin de produire des œufs et du fromage frais. Les parterres surélevés rendent néanmoins 
compte des difficultés à cultiver la terre sur des parcelles disposant d'un sol relativement maigre et parfois pollué. Les bénéfices ne se limitent toutefois pas à des enjeux alimentaires : la réappropriation d'espaces vacants favorise la reconstitution d'un capital social de proximité, dans des quartiers où les liens sociaux se sont afFaiblis dans un contexte de déclin urbain. À l'arrière-plan, le bâtiment en brique abrite un centre communautaire équipé d'ordinateurs et d'une bibliothèque, destinés aux loisirs des enfants et jeunes du quartier.

Figure 3. Jardin communautaire du Georgia Street Community Collective (East Side, Detroit, MI)

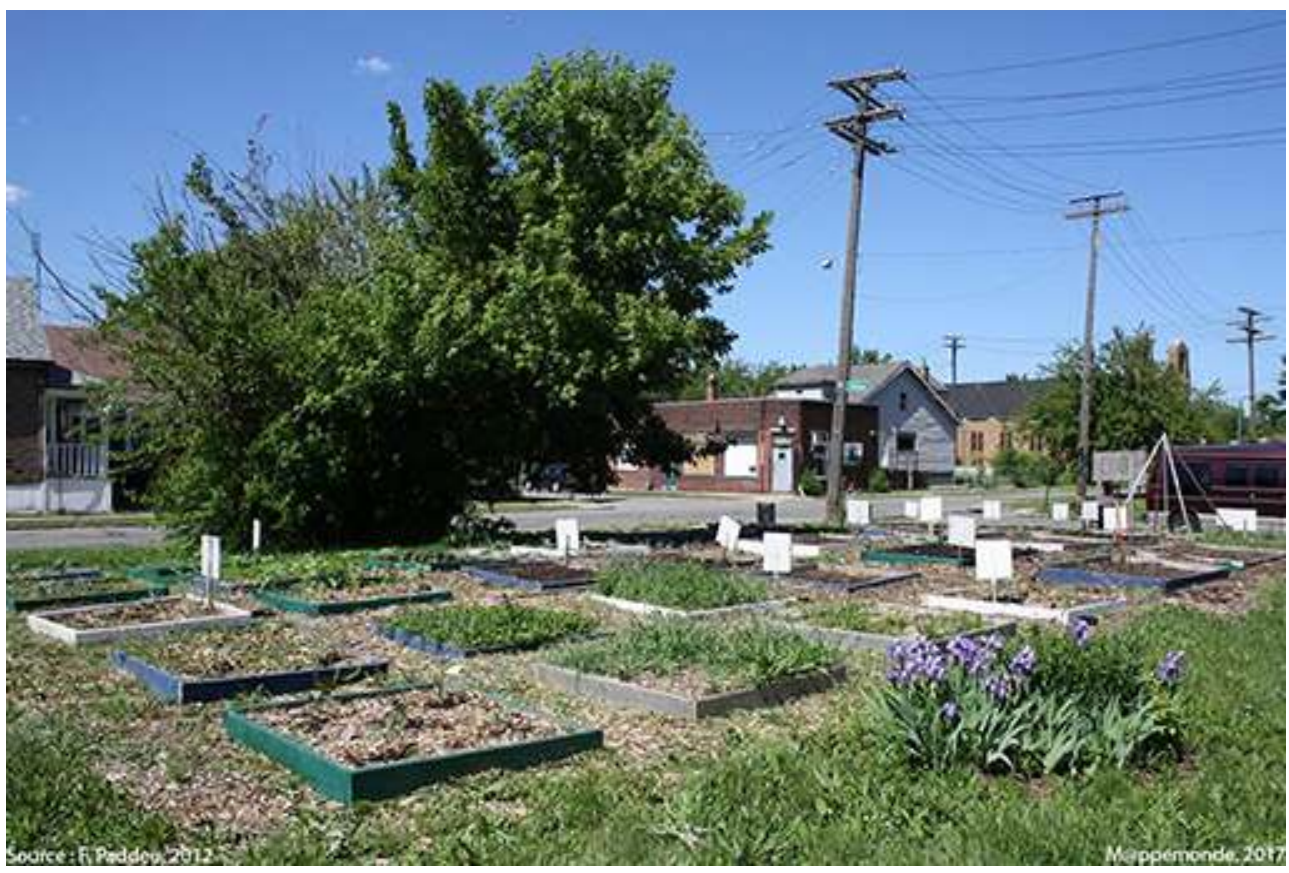

Source : F. Paddeu, 2013

L'enquête a ainsi montré que la réappropriation des espaces vacants constitue un dispositif novateur pour ces mobilisations en quartier en déclin. Alors que les parcelles vacantes étaient traditionnellement perçues comme un stigmate de la crise urbaine, un symbole du traumatisme, du déclin et une source de désordres matériels et sociaux, elles sont désormais envisagées comme une opportunité pour expérimenter de nouvelles pratiques écologiques et agricoles (Hollander et al., 2009), créer des « lieux communs » et lutter contre les efFets négatifs de la vacance foncière. Dans un contexte de vacance du pouvoir politique et à l'heure où une parcelle coûte 500 dollars à Detroit, j'ai montré que cela n'en reste pas moins une opportunité ambiguë, au regard des risques encourus en termes de spéculation foncière ou de land grabbing. 


\section{BIBLIOGRAPHIE}

DETROIT WORKS PROJECT LONG-TERM PLANNING STEERING COMMITTEE , (DETROIT WORKS). (2012). Detroit Future City. Detroit Strategic Framework Plan. Detroit, 347 p.

DUCHEMIN É., WEGMULLER F., LEGAULT A.-M. (2010). « Agriculture urbaine : un outil multidimensionnel pour le développement des quartiers ». Vertigo, vol. 10, n², p. 1-10. https:// journals.openedition.org/vertigo/10436 HOLLANDER J.B., PALLAGST K.M., SCHWARZ T., POPPER F.J. (2009). « Chapter 4. Planning Shrinking Cities ». In BLANCO HILDA, ALBERTI Marina (dir.), « Shaken, Shrinking, Hot, Impoverished and Informal: Emerging Research Agendas in Planning », Progress in Planning, vol. 72, n 4 p. 223-232. SKogAn W.G. (1990). Disorder and Decline: Crime and the Spiral of Decay in American Neighborhoods. New York: The Free Press, 218 p.

SUGRUE T.J. [1996] (2013). The Origins of the Urban Crisis: Race and Inequality in Postwar Detroit. Princeton : Princeton University Press, $375 \mathrm{p}$

INDEX

Mots-clés : prix de thèse, prix de thèse 2016 